\title{
Féeries
}

Études sur le conte merveilleux, XVII $-\mathrm{XIX}{ }^{\mathrm{e}}$ siècle

9 | 2012

Le dialogisme intertextuel des contes des Grimm

\section{Les jeux de la reconfiguration dans Schneewittchen de Robert Walser}

Games of reconfiguration in Robert Walser's Schneewittchen

\section{Pascale Auraix-Jonchière}

\section{(2) OpenEdition}

1 Journals

\section{Édition électronique}

URL : http://journals.openedition.org/feeries/825

DOI : $10.4000 /$ feeries.825

ISSN : 1957-7753

Éditeur

UGA Éditions/Université Grenoble Alpes

\section{Édition imprimée}

Date de publication : 15 octobre 2012

Pagination : 113-138

ISBN : 978-2-84310-233-2

ISSN : 1766-2842

\section{Référence électronique}

Pascale Auraix-Jonchière, "Les jeux de la reconfiguration dans Schneewittchen de Robert Walser », Féeries [En ligne], 9 | 2012, mis en ligne le 15 octobre 2013, consulté le 07 septembre 2020. URL: http://journals.openedition.org/feeries/825 ; DOI : https://doi.org/10.4000/feeries.825

\section{(c) Féeries}




\section{LES JEUX DE LA RECONFIGURATION DANS SCHNEEWITTCHEN DE ROBERT WALSER}

$B$ LANCHE-NEIGE (SCHNEEWITTCHEN) fait partie des "dramolets féeriques» que Robert Walser publie dans la revue Die Insel entre I900 et I902, ainsi que Cendrillon (Aschenputtel). Les deux ouvres de jeunesse se présentent comme des récritures des contes des frères Grimm. Plus tard, Walser reprendra sous plusieurs formes l'histoire de «La Belle au bois dormant». Un petit drame, Dornröschen, date de 1920 ; une courte prose figure dans le recueil Vie de poète.

Le drame lyrique de Walser participe doublement de l'esthétique de son temps. Comme l'a montré Peter Utz, les dramolets ne sont pas dissociables de la "discussion contemporaine autour du renouvellement dramatique» qui vise à remplacer «le drame classique» par «le drame psychique» dans lequel les héros, souvent confrontés à l'idée de leur propre fin, "se délivrent de leurs rêves et de leurs souvenirs par le discours ${ }^{2}$ ". Mais il rejoint aussi les nombreux auteurs européens qui, à la fin du $\mathrm{XIX}^{\mathrm{e}}$ siècle et contre toute attente, font retour sur les contes — de Perrault notamment ${ }^{3}$ — pour produire chacun à leur façon ce que Jean de Palacio a nommé «perversions du merveilleux ${ }^{4} »$.

La didascalie inaugurale situe le drame après le mariage de celui que les frères Grimm nommaient «fils de roi » (Königssohn) ${ }^{5}$ et de la Princesse, tout

I. Carouge-Genève, Éd. Zoé, 2006.

2. P. Utz, Robert Walser : danser dans les marges, 200I, p. 24. Voir les exemples de Mallarmé, de Maeterlinck ou de Hoffmansthal.

3. «À l'examen, Grimm [...] se révélait infiniment moins invoqué que Perrault, jouissant d'une notoriété moindre. [...] Et ceci ne valait pas que pour la France. En Angleterre, c'est à des modèles français et perraltiens que recouraient Thackeray ou Dickens dans leurs réécritures. [...] Avec Perrault, c'était l'ensemble d'une œuvre qui était pris en compte comme ferment ou levain d'un nouveau merveilleux.» (J. de Palacio, Les Perversions du merveilleux, 1993, p. 2I-22.)

4. Titre de l'ouvrage précédemment cité.

5. Contes pour les enfants et la maison: collectés par les frères Grimm, édités et traduits par N. Rimasson-Fertin, 2009, t. I, p. 303. La version originale, en allemand, est consultable sur le site 
en éludant le terrible dénouement du conte, qui montre la Reine impie et jalouse en train de danser, chaussée de "souliers de fer» (eiserne Pantoffeln) rougis au feu "jusqu'à ce qu'elle s'effondre, morte, sur le sol» (Da mußte sie [...] so lange tanzen, bis sie todt zur Erde fiel) ${ }^{6}$. En effet, sont présents "la Reine, Blanche-Neige, le Prince étranger, le Chasseur» (Die Königin, Schneewittchen, der fremde Prinz, der Jäger) ${ }^{7}$. Ce personnel postule en soi une reconfiguration d'ensemble puisque la Reine n'a pas été soumise au châtiment cruel et moralisateur sur lequel se clôt le conte des frères Grimm et que le Chasseur, de personnage secondaire (qui disparait une fois qu'il a feint de remplir sa mission), devient personnage pérenne. Il s'agit bien pourtant du conte de référence, celui dont Blanche-Neige rappelle une première fois la trame obsédante dans sa première tirade, comme les déterminants définis l'attestent. "La Reine», «le Prince» et "le Chasseur», non loin de "l'entrée $d u$ château " $(\text { das } S c h l o \beta)^{8}$, renvoient clairement au conte. Ce château déjà connu du lecteur est à n'en point douter celui où eut lieu le splendide mariage : "Viens avec moi au château de mon père, tu seras mon épouse" (komm mit mir in meines Vaters $S c h l o \beta){ }^{9}$, dit le fils de roi à Blanche-Neige tout juste sortie du cercueil. La continuation du conte repose par conséquent sur un ensemble de modifications qui semblent devoir redistribuer les forces en présence. Le choix d'un lieu inédit en est la preuve discrète : la scène se situe dans « un jardin » (ein Garten), nullement mentionné chez les Grimm où, à l'intérieur des châteaux et de la "petite maisonnette» (ein kleines Häuschen) des sept nains, s'oppose le dehors de la "grande forêt» (in dem großen Wald) ${ }^{\text {10}}$. Espace socialisé donc, pour un entretien et une confrontation qui, renvoyant au dit du conte, n'ont de cesse de le commenter et de le recréer.

Dans les récritures fin-de-siècle, Jean de Palacio distingue trois «modalités de la perversion ${ }^{\mathrm{II}}$ » : "la perversion par suite», «la perversion par extension » (qui se focalise sur un objet ou un détail), "la perversion par contrefaçon » (qui consiste en une plongée du conte dans la modernité) ${ }^{12}$. Il remarque que la première catégorie, dont semble faire partie le dramolet de Robert

<http://de.wikisource.org/wiki/Schneewittchen>, suivi de la date de l'édition choisie et du numéro de page.

6. Contes pour les enfants et la maison, ouvr. cité, p. 304.

7. R. Walser, Blanche-Neige, 2006, p. 9.

8. Ibid.

9. Contes pour les enfants et la maison, ouvr. cité, p. 304.

IO. R. Walser, ouvr. cité, p. 297.

II. J. de Palacio, ouvr. cité, p. 29.

I2. Ibid., p. 38, 42, 44. 
Walser, est «attentatoire au texte initial» dont la clôture est mise à mal en même temps qu'est fissurée sa cohérence ${ }^{\mathrm{I} 3}$. Mais chez Walser, le mécanisme sur lequel repose la récriture est plus complexe puisque l'un des enjeux en est le changement de genre. On note, par ailleurs, que le conte ne subit pas le transfert, facteur de prosaïsme, qu'implique la fréquente " contrefaçon ${ }^{\mathrm{I}}$ », ici absente, et le léger changement d'espace (le jardin) ne brise pas l'uchronie si particulière au genre. Pourtant le "seuil formulaire ${ }^{15}$ " «il était une fois " sur lequel s'ouvre le conte de Grimm n'est plus présent comme marqueur de "distanciation ${ }^{16} »$. Mais l'ouverture in medias res, conjuguée aux déterminants définis à valeur quasi anaphorique (dans le sens où ils renvoient à un avant-texte qui s'impose clairement comme référent), a pour effet de maintenir la scène dans cet espace-temps singulier qui nous fait assister à l'action qui «est devant nous, à distance ${ }^{17}$ ». Lorsqu'il repense ce dispositif, Jean-Michel Adam conclut à une «localisation [du conte] dans un monde de langage ${ }^{18} »$. Dans le cas présent, la mise en vers et la mise en scène transforment de fait le texte en un ballet verbal dont l'objet principal serait la matière même du conte merveilleux. Loin de provoquer un décrochage par rapport à l'éloignement inhérent au genre, la pseudo-continuation de Walser, que Dieter Borchmeyer a qualifiée à juste titre de "métathéâtre ${ }^{19}$ ", intensifie donc le phénomène de présentation distanciée propre au conte. C'est un espace langagier au carré qui nous est en fait présenté et qui, faisant retour sur le conte plus qu'il ne le déroule, en déconstruit le sens sans pour autant le dégrader. Dans ce jeu théâtral, l'ensemble des personnages se donne en représentation. Le jeu scénique renvoie ainsi à un drame de l'intériorité qui se déploie au fil de ses réflexions sur les séquences et sur le sens du conte, qui se réinvente à mesure.

13. Ibid., p. 39.

I4. Le conte alors «intègre l'actualité des soucis de la fin du siècle» et «suscite des préoccupations, des paroles et des décors opposés à la logique du conte» (ibid., p. 44).

I5. J.-M. Adam, «Fiction et narration», dans U. Heidmann et J.-M. Adam, Textualité et intertextualité des contes, 20IO, p. 234.

16. Ibid., p. 24I.

17. Jean Pouillon, Temps et roman, Paris, Gallimard, 1946. Cité par J.-M. Adam, art. cité, p. 24I.

I8. Ibid., p. 24I.

19. Dieter Borchmayer, «Robert Walsers Metatheater. Über die Dramolette und szenischen Prosastücke», dans P. Chiarini et H. D. Zimmermann (éds), „Immer dicht vor dem Sturze... “. Zum Werk Robert Walsers, Francfort-sur-le-Main, 1987, p. I29-I43. Cité par P. Utz, ouvr. cité, p. 48. 


\section{Brouillages}

Le drame se joue d'abord entre Blanche-Neige et la Reine, sur fond de rivalité funeste :

Königin:

Sag', bist du krank?

Schneewittchen:

Was mögt Ihr fragen, da Ihr doch

den Tod wünscht der, die als zu schön

Euch immer in die Augen stach.

[...]

Ich bin nicht krank; ich bin ja tot.

La Reine :

Dis, tu es malade?

Blanche-Neige :

Quelle question, quand vous n'avez que vœux de mort pour la trop belle qui blesse à tout instant vos yeux.

[...]

Malade, moi? Non, je suis morte ${ }^{20}$.

On reconnaît là le schéma fondateur, qui dans le conte oppose à l'enfant grandissant en beauté une figure maternelle négative, la seconde épouse du père, que les frères Grimm désignent ${ }^{21}$ assez sobrement par une courte série de syntagmes essentiellement dépréciatifs. La Reine — tel est le terme dominant - est appelée "marâtre» (Stiefmutter) à plusieurs reprises ${ }^{22}$. Le terme renvoie d'une part à un statut civil (la marâtre est bien la seconde femme du père, qui a des enfants d'un premier lit), d'autre part à des connotations péjoratives, qui évoquent sa cruauté ( Femme qui maltraite les enfants que son mari a eus d'un mariage précédent ", précise le Trésor de la langue française). Le premier paragraphe du conte, que l'on peut qualifier de génésiaque, s’achève en effet par la mort de la mère biologique : «Et

20. R. Walser, ouvr. cité, p. 9.

2I. "Une belle femme» (eine schöne Frau), p. 296; "cette méchante femme», p. 297 ou «la méchante femme» (das böse Weib), p. 300 ; "cette femme perfide», p. 301 ; «la marâtre impie» (gottlose Stiefmutter) et "cette femme perfide», p. 304.

22. Voir la traduction de N. Rimasson-Fertin, par exemple à propos du mariage du Prince et de Blanche-Neige : "Cependant, la marâtre impie de Blanche-Neige fut aussi invitée à la fête», p. 304. La version allemande donne : "Zu dem Fest wurde aber auch Sneewittchens gottlose Stiefmutter eingeladen". 
aussitôt après la naissance de l'enfant, la mère mourut.» (Und wie das Kind geboren war, starb die Königin.) $)^{23}$

Or Walser met en place tout un paradigme de la filiation qui a pour effet de brouiller la désignation et de jeter un doute sur le texte de référence. Dans la bouche des personnages, essentiellement la Reine et Blanche-Neige, le lien mère-fille devient un leitmotiv et joue sur l'ambiguïté de leurs statuts respectifs. Le premier écart par rapport au texte source est la fréquence de l'association «mère/enfant». Dès l'ouverture du drame, Blanche-Neige emploie le vocatif «Mère" (Mutter) et la Reine en retour celui de "gracieuse enfant» (Hold Kind) ${ }^{24}$. Le rapport parental devient donc central dans cette récriture, qui multiplie les emplois de ce binôme lexical (mère/ enfant). Toutefois, le statut de la Reine fait problème : s'agit-il toujours de la marâtre, ou bien plutôt de la mère naturelle? Si à cet égard le substantif "mère" reste équivoque, il n'en va pas de même de "maman", dont on relève plusieurs occurrences : «[...] ne puis-je donc saluer Maman / qui en bas dans l'ombre du parc / s'occupe à des travaux d'aiguille?" (Ist es mir nicht erlaubt / durch dich zu grüßen die Mama, I die unten in dem schatt'gen Park I mit Stickereien beschäftigt ist? ${ }^{25}$, demande Blanche-Neige. Le glissement sémantique de "Mutter» à "die Mama», que redouble le rappel de la scène initiale du conte dans laquelle, de fait, c'est bien la mère qui coud et rêve à sa fenêtre, puis émet un vœu qui lui permettra d'enfanter, suppose une distorsion du récit de référence, à moins qu'il ne s'agisse d'un renvoi à la première édition des contes de Grimm, celle de I8I2. En effet, dans cette première version, la mère ne meurt pas et c'est bien elle, la plus belle du pays, qui nourrit jalousie et aversion envers sa propre fille dès lors que celle-ci, à l'âge de sept ans, est déclarée supérieure en beauté par le miroir magique $^{26}$. Le substantif «maman» (Mama) est encore employé dans une adresse directe de la Princesse à la Reine ${ }^{27}$. Mais si, de son côté, la Reine emploie le terme d' "enfant» (Kind) pour désigner Blanche-Neige, c'est toujours en ménageant une forme de distanciation suspecte.

Cette mise à distance, qui pourrait faire penser que cet emploi est figuré plus que dénotatif - et que, par conséquent, il n'existe pas de lien de sang entre mère et fille —, se traduit de trois façons. D'abord, le binôme

23. Contes pour les enfants et la maison, 2009, t. I, p. 296.

24. R. Walser, ouvr. cité, p. Io.

25. Ibid., p. 4I-43.

26. L'édition de I8I2 donne pour le dénouement : "Auf den andern Tag war die Hochzeit bestellt, und Sneewittchens gottlose Mutter, auch eingeladen."

27. R. Walser, ouvr. cité, p. 52-53 : "D’un baiser du prince, ne dites / plus mot, Maman.» (Von einem Prinzenkusse sagt / nichts mehr, Mama.) 
«enfant» / «mère» ou «enfant» / «parents» apparaît dans des aphorismes ou des énoncés assimilables : «Beauté en son enfant est baume / pour les désirs las de la mère" (Schönheit am eignen Kinde ist / Balsam für müde Mutterlust ${ }^{28}$; "Va, consens, sois l'enfant qu'on aime, / crois les parents comme toi-même" (Wend dich nicht ab, sei liebes Kind, I trau Elternwort als wie dir selbst ${ }^{29}$. Le second exemple combine tournure universalisante et injonction, ce qui a pour effet de mettre à distance le couple parents/enfants et à l'inverse d'actualiser et d'exemplifier le schéma relationnel idéal proposé en référence. À cette tournure généralisante, qui ne donne pas vraiment la clé du texte (s'agit-il là d'un simple modèle convoqué par la Reine?) s'ajoute l'emploi de comparaisons qui semblent remettre en cause (ou refuser) l'idée de maternité. «Je t’aime / comme l'enfant de ma chair même!» (Wie hättich Ursach', Grund und Recht / zu hassen dich, die mir so lieb / als wie das Kind der eignen Brust!' $)^{30}$, s'exclame la Reine. Le comparatif ne s'explique que comme la projection d'un lien qui ne correspond pas à la réalité. Il en va de même de cette autre déclaration, quoique sur un mode plus atténué : "Vrai, j’ai toujours aimé en toi / mon enfant chéri, sans péché» (Vielmehr ich liebte immer dich / als mein unschuldig liebes Kind) ${ }^{31}$. Si la Reine aime Blanche-Neige comme son enfant, c'est qu'elle n'est pas sa mère, ou qu'elle repousse loin d'elle cette idée. Et corrélativement, si Blanche-Neige appelle la Reine «maman », c'est que celle-ci est bel et bien sa mère, ou alors qu'elle emploie ce terme affectueux dans un sens ironique - interprétation qu'autorise le contexte walsérien. Un dernier procédé consiste à créer un double effet d'étrangeté et de proximité par le biais d'une tournure attributive ou d'un complément circonstanciel :

Schneewittchen, komm. Prinz, Ihr erlaubt,

ich nenne sie mein herzig Kind.

[...] Viens, Blanche-Neige.

Avec votre permission, Prince,

je la nomme enfant de mon cœur ${ }^{32}$.

L'expression "enfant de mon cœur» (mein herzig Kind) ne figure pas en emploi direct. Le relais («je la nomme», «ich nenne sie»), ici redoublé par la situation d'énonciation (l'adresse au Prince), la rend artificielle — sinon artificieuse. On trouve un emploi quasi équivalent dans les propos de

\footnotetext{
28. Ibid., p. I8-I9.

29. Ibid.

30. Ibid., p. I8-I9.

3I. Ibid., p. I6-I7.

32. Ibid., p. 70-7I.
} 
Blanche-Neige lorsque celle-ci accorde son pardon à la Reine, alors désignée comme "la pécheresse / dont, en loyale enfant, je baise / la main» (Der Sünd'rin hier / küss' ich als treues Kind die Hand) ${ }^{33}$. Le circonstanciel peut se comprendre comme une comparaison implicite, une sorte de jeu de rôle. Le jeu scénique précisément autorise ces fluctuations sémantiques et les rend sensibles. Si en effet tout est jeu, comme le déclarent à maintes reprises les protagonistes, pourquoi ne pas jouer aussi des relations entre les personnages, y compris celle, fondamentale, de filiation? Ce parti pris renvoie au trouble de Robert Walser devant l'instabilité du signifié. À ses yeux, les apparences, toujours, sont trompeuses : «la mère n'est pas la mère» (die Mutter ist die Mutter nicht), affirme Blanche-Neige ${ }^{34}$. Konrad Harrer commente : «Si bien que chez Walser la marâtre devient le symbole même des fausses apparences et de la rupture entre signifiant et signifié35. " Mais cette rupture introduit aussi un flottement interprétatif quant aux principes de la récriture du conte : est-il bien sûr qu'il s'agit toujours de la marâtre, comme l'affirme plus clairement encore Fabienne Raphoz-Fillaudeau dans son édition du texte de Walser ${ }^{36}$ ? Et ne peut-on avancer que l'écrivain lui aussi joue sur la double partition des versions du conte?

\section{Déploiements par métaphorisation}

Les principaux motifs qui structurent le conte se retrouvent disséminés dans le dramolet, ne serait-ce qu'à l'occasion des séquences rétrospectives qui relient clairement cette "suite» au récit des frères Grimm, dont elles rappellent le contenu. Trois d'entre eux sont privilégiés et font l'objet d'un certain nombre de transferts ou amplifications : l'animal sacrifié, la pomme empoisonnée, la neige.

L'épisode du chasseur, qui donne lieu à plusieurs récritures, prenant par là une ampleur considérable, est l'occasion d'une légère distorsion. Dans le conte le Chasseur, qui prend pitié de Blanche-Neige, tue «un jeune marcassin" qui "passait justement par là» (ein junger Frischling daher gesprungen kam) pour en rapporter à la Reine, qui les mange, «les poumons

33. Ibid., p. IO2-IO3.

34. Ibid., p. 78-79.

35. K. Harrer, Souveraineté et impuissance dans l'ouvre de Robert Walser, 2008, p. 273.

36. «Que s'est-il passé avant la réconciliation présumée de Blanche-Neige et de sa belle-mère?» (R. Walser, ouvr. cité, «Dossier complémentaire», p. II5.) 
et le foie» ([...er] nahm Lunge und Leber heraus, und brachte sie [...] der Königin mit) ${ }^{37}$. Le chasseur du dramolet quant à lui répète ainsi la scène :

Mich rührte Eure holde Bitt',

Eur Angesicht, süß wie der Schnee,

der unterm Kuß der Sonne liegt.

Ich steckte ein, womit ich Euch

ermorden wollte, stach das Reh,

das eben durch die Quer uns sprang,

sog gierigen Sinns das Blut aus ihm.

Votre grâce à prier m'émut,

et votre visage aussi doux

que neige au baiser du soleil.

Je rempochai ce avec quoi

j'allais vous tuer, je poignardai

la biche qui vint à traverse,

tirai avidement son sang ${ }^{38}$.

Mich rührte gleich von Anfang an

die flehnde, süße Kindesbitt',

die dir aus Mund und Auge sprach.

Ich senkte Dolch und Arm zugleich

und hob dich, Süße, zu mir auf.

Das Reh, das in die Quer uns sprang,

erstach ich mir. Ist es nicht so?

Je fus sur-le-champ touché par

la douce prière d'enfant

montant de ta bouche et de tes yeux.

J'abaissai d'un coup dague et bras,

je t'élevai, douce, vers moi.

La biche jetée à traverse,

je l'égorgeai. N'est-il pas vrai ${ }^{39}$ ?

Le résumé du conte, qui préserve les articulations majeures et l'esprit de la séquence, qu'elle teinte cependant d'un discret érotisme, introduit curieusement un changement de détail : au «jeune marcassin» (ein junger Frischling) se substitue la biche (das Reh). Cette modification apparemment secondaire est d'autant plus insolite que, si l'on en croit l'index thématique établi par Natacha Rimasson-Fertin sur la base de celui de Hans-Jörg Uther, l'animal n'est jamais mentionné dans l'édition de 1857 des Kinder-und

37. Contes pour les enfants et la maison, 2009, t. I, p. 297.

38. R. Walser, ouvr. cité, p. I2-I3.

39. Ibid., p. 92-93. 
Hausmärchen. La version de I8I2 ne diffère pas non plus sur ce point. Or, dans une troisième occurrence, l'animal prend un sens figuré pour qualifier le prince dont les sens sont troublés par la Reine :

Schneewittchen:

[...]

Welch eine Meute Hunde reizt

dir so den Sinn, daß wie das Reh

erschrocken du dem Feinde fliehst,

der dich verfolgt.

Blanche-Neige :

[...]

Quelle meute de chiens t'excite

les sens au point que tu fuies, telle

la biche effrayée, l'ennemi

qui te poursuit ${ }^{40}$.

Le motif reste assez convenu : la biche traquée fonctionne a priori à la manière d'un cliché, mais le maillage des images est tel dans le drame que l'on est tenté de rapprocher les trois occurrences. La comparaison a pour effet de féminiser le prince et d'insister sur sa fragilité et son côté versatile. C'est dire qu'il ne saurait correspondre au rôle établi par la tradition, et ces fluctuations traduisent la propension du personnage à échapper à la gangue sémantique qui l'emprisonne. Mais au-delà, un discret réseau de correspondances se met en place. En effet, le prince chez Walser n'est qu'un jeune garçon instable, plus proche en cela de la version de I8I2 où il est précisé : "Einmal kam ein junger Prinz zu dem Zwergenhaus ${ }^{4 \mathrm{1}}$ ", tandis que l'adjectif disparaît dans les éditions postérieures. La Blanche-Neige de Walser le décrit ainsi :

er hat ja noch kein Haar am Kinn,

er ist ein kleiner Knabe noch,

sonst edel, aber furchtbar klein,

schwach, wie der Leib, worin er steckt,

klein, wie der Sinn, woran er hängt.

Il n'a pas de poil au menton,

c'est encore un petit garçon,

noble, oui, mais diantrement petit,

chétif quant au corps où il loge,

petit, quant au sens qui est sien ${ }^{42}$.

40. Ibid., p. 40-4I.

4I. Kinder-und Hausmärchen, Wikisource, édition de I8I2.

42. R. Walser, ouvr. cité, p. 52-53. 
Cette jeunesse, rendue extrême et ridicule par Robert Walser, présente le prince comme l'antonyme du chasseur viril que le drame convertit en amant de la Reine et dont il observe, en voyeur, les exploits amoureux ${ }^{43}$. Dès lors, la biche sacrifiée par la main du chasseur, amant aux «bras puissants " (starken Arm), "coquin» sans «nulle pudeur» (Der Schurke ist ganz unverschämt $)^{44}$, pourrait renvoyer l'image du jeune prince inexpérimenté, réduit à néant par le spectacle perturbant, "comme flot trouble d'eau boueuse" (wie die schlammige Flut) et "double à voir " du couple aimant (Ach, 's ist ein / zwiefacher Anblick) ${ }^{45}$. La distorsion, pourtant légère, crée une forme de clinamen qui provoque la redistribution nouvelle de motifs qui essaiment pour faire ricochet sur le sens.

Mais le ricochet peut prendre diverses formes. Dans l'exploration à rebours du conte à laquelle procèdent les personnages, les tentatives de la Reine pour tuer Blanche-Neige se concentrent sur deux épisodes corrélés : le sacrifice du chasseur et le don de la pomme empoisonnée. Celui-ci correspond au troisième et dernier essai de la souveraine pour éliminer sa rivale. Sous l'apparence d'une vieille femme, elle lui propose d'abord un lacet pour serrer son corset, puis un "peigne empoisonné» ([Sie machte] einen giftigen Kamm), enfin "une pomme très, très empoisonnée" (einen giftigen, giftigen Apfel) ${ }^{46}$. Robert Walser, qui retient le poison comme dénominateur commun (dans un sens littéral et figuré), privilégie la pomme. Mais, partant de la lettre du conte, il en redéploie là encore le sens. Car la pomme concentre le mal dont souffrent tous les personnages, comme le proclame Blanche-Neige dès l'ouverture de la pièce : "La pomme empoisonnée fait mal, / oh, oh, si mal, et de vous, Mère, / c'est de vous que je l'ai reçue» (Der gift'ge Apfel tat so weh, I o, o, so weh, und, Mutter, Ihr, I Ihr seid es, die ihn mir gebracht ${ }^{47}$. Là encore, le dit du conte est contenu dans le drame sous forme de rappel : «Pomme et poison venaient de moi : / tu y goûtas, tu en mourus" (Den gift'gen Apfel schicktich dir; / du aßest ja davon und starb), résume la Reine ${ }^{4}$. De son côté, le Prince amplifie cet épisode dans un métadiscours qui dénonce l'action et convoque la structure polémique afférente au conte : «Du poison, comme à un chien, fut /

43. La Reine apostrophe le Prince par la formule dépréciative «petit prince» (kleiner Prinz), p. 2I, à quoi ce dernier répond : «Bien sûr, j'ai l'air petit et faible» (Jawohl, und schein'ich klein und schwach), p. 23.

44. R. Walser, ouvr. cité, p. 32-33.

45. Ibid., p. 30-3I.

46. Kinder-und Hausmärchen, édition de 1857.

47. R. Walser, ouvr. cité, p. IO-II.

48. Ibid., p. 50-5I. 
donné à cette douce enfant " (Gift wurde diesem süßen Kind vorgestreut) ${ }^{49}$. En effet, le contraste entre la comparaison et la collocation «douce enfant » (süßen Kind) introduit subrepticement un commentaire sur le conte. Le geste devient ouvertement condamnable et le conte merveilleux s'apparente aux histoires tragiques dont il n'est pas si loin ${ }^{50}$. Le dramolet fait en outre intervenir de façon explicite le concept de péché : les simples catégories du Bien et du Mal dont le conte articule la confrontation prennent en effet une coloration religieuse chez Walser. Blanche-Neige appelle la Reine «pécheresse aimée» (der lieben Sünderin) ${ }^{\text {sI }}$, et s'efforce d'inverser le sens de l'histoire :

[...] Mein Gefühl

spricht Euch von aller Sünde frei,

liegt auf den flehnden Knien vor Euch

und bittet, nennt mich Sünderin,

mich, die so bang Verzeibung fleht.

De tout péché mon sentiment

vous lave, à genoux, il implore,

prie : appelez-moi pécheresse,

j'implore si fort le pardon ${ }^{52}$.

L'aveu de la Faute ("Pomme et poison venaient de moi»; «Den gift'gen Apfel schickt' ich dir») constitue la réponse de la Reine à cette tirade. On peut donc supposer que la focalisation du dramaturge sur la pomme, parmi les trois objets utilisés par la Reine pour tuer Blanche-Neige dans le conte des frères Grimm, s'explique par cette dérivation religieuse du propos ${ }^{53}$. En même temps, ce glissement a pour finalité paradoxale d'évacuer de la pièce toute notion de culpabilité et de remords. Susciter l'idée de péché pour le vider de son sens, tel serait l'objectif de Robert Walser en réactivant, via le conte, le motif biblique lié à la Faute originelle. Lorsque, dans un autre épisode, Blanche-Neige accuse la Reine d'avoir voulu sa mort et que cette dernière feint d'adhérer à cette version — qui est bien celle que propose le conte de référence —, la Princesse l'interrompt en ces termes :

49. Ibid., p. 22-23.

50. Voir sur ce point J.-M. Adam, dans U. Heidmann et J.-M. Adam, Textualité et intertextualité des contes, p. 247 : l'histoire tragique est «fort à la mode surtout dans la première moitié du $\left[\mathrm{XvII}^{\mathrm{e}}\right]$ siècle et $[\ldots]$ on peut [la] considérer comme une forme de littérarisation des canards sanglants proches de nos modernes faits divers".

5I. R. Walser, ouvr. cité, p. 52-53.

52. Ibid., p. 50-5I.

53. Cette christianisation se traduit par l'introduction de tout un ensemble de motifs messianiques — notamment l'apparition récurrente de l'image du cerf, associée à saint Hubert — dans le film des frères Quay, Institut Benjamenta, inspiré des écrits de R. Walser. 
Genug, genug, es macht Euch toll.

Hätt'ich die gift'ge Wunde doch

nicht mehr berührt. Nun blutet sie

frisch wieder und wird nimmer heil.

Wenn Ihr verzeihtet, Königin.

Assez, assez, ça vous rend folle.

Pourquoi ai-je touché la plaie

empoisonnée? Voilà, rouverte,

qu'elle saigne, et ne peut guérir.

Reine, sauriez-vous pardonner ${ }^{54}$ ?

La «plaie empoisonnée» (die gift'ge Wunde), associée à la notion de pardon et donc à celle de péché, renvoie à la pomme du conte par métonymie et à son exploitation dans le drame par métaphore. Pour autant ce n'est pas le crime qui est ainsi désigné, mais l'idée que l'on s'en fait. Nous touchons là à l'une des modalités de la reconversion du conte opérée par Walser, qui s'oppose à l'univocité jugée sclérosante qu' induit le dispositif de ce dernier. C'est bien le même terme (giftig) qui est employé par Walser dans ce passage et qu'utilisent les frères Grimm pour qualifier le fruit vénéneux ${ }^{55}$. Mais le dramaturge en explore le spectre sémantique et l'emploie dans un sens là encore figuré. Le chasseur explique :

Die Königin schikte nicht nach dir

mit Gift zu deinen Zwergen aus.

Der gift'ge Apfel ist nicht wahr.

Die Lüg' ist giftig, die das sagt.

Sie selbst, die das behauptet, ist geschwoll'n wie eine schöne Frucht, verlockend und voll Schmeichelpracht, doch innen so, daß krank wird, wer davon zu kosten sich erkünnt.

La Reine jamais ne te fit porter du poison chez tes nains. Pomme et poison, rien qui soit vrai. Rien qu'un mensonge empoisonné qui s'emplit de ses propres dires pour se gonfler comme un beau fruit tentateur, plein de séduction mais tel, au-dedans, qu'à oser y goûter, on tombe malade ${ }^{56}$.

54. R. Walser, ouvr. cité, p. 8o-83.

55. L'expression reste inchangée d'une édition à l'autre.

56. R. Walser, ouvr. cité, p. 92-95. 
Ces propos attestent la corrélation «pomme-poison-péché», qu'ils détournent dans le même temps de son sens initial : une nouvelle extension métaphorique sert à désigner le dit du conte et à en réfuter l'autorité. La séquence de la pomme, que désigne la périphrase, change de catégorie comme de signification : le récit se fait image pour dénoncer le mode de fonctionnement du conte merveilleux, redéfini comme une construction fascinante mais close sur elle-même. C'est précisément à cette clôture du sens et à ses présupposés moraux que le poète dramaturge tente d'échapper.

Enfin, un même discours figuré se développe à propos du personnage éponyme autour du motif de la neige. Pourtant, s'il est bien matriciel dans le conte, il n'est employé qu'à deux reprises en dehors du paragraphe inaugural, et encore n'est-ce que sous forme de reprise littérale, la mort supposée de Blanche-Neige faisant écho par deux fois à sa naissance. L'incipit amorce une rêverie élémentaire sur la neige, et ne contient pas moins de six occurrences du substantif, sans compter celle du nom composé de l'héroïne. L'évocation, qui trouve vraisemblablement sa source dans le Perceval de Chrétien de Troie, est essentiellement liée au désir, désir de beauté, de perfection et de pureté : le personnage de Blanche-Neige, dont cette ouverture semble avoir pour fonction essentielle d'expliquer le nom, sera portraituré une fois pour toutes; c'est «une petite fille aussi blanche que la neige, aussi rouge que le sang et qui avait les cheveux aussi noirs que l'ébène" (ein Töchterlein, das war so weiß wie Schnee, so roth wie Blut, und so schwarzhaarig wie Ebenholz) ${ }^{57}$. Plus tard, quand la Reine, qui l'a empoisonnée, la contemple, elle emploie la formule de façon ironique ${ }^{58}$ et lorsque les nains la déposent dans son cercueil de verre, "elle était toujours aussi blanche que la neige, aussi rouge que le sang et ses cheveux étaient toujours aussi noirs que le bois d'ébène" (es war noch so weiß als Schnee, so roth als Blut, und so schwarzhaarig wie Ebenholz) ${ }^{59}$. Entre temps, cependant, aucune mention de la neige n'apparaît. Le dramolet, au contraire, exploite les valeurs symboliques du motif, emprunté à l'incipit du conte ici absent. Comparaisons et métaphores se multiplient pour définir la figure centrale de Blanche-Neige, mélange de séduction et de froideur. Le Chasseur se laisse prendre à ce charme si particulier, qui fléchit sa cruauté : "Votre grâce à

57. Contes pour les enfants et la maison, 2009, t. I, p. 296.

58. "La Reine la contempla alors d'un air mauvais puis elle se mit à rire à gorge déployée en disant: "Blanche comme la neige, rouge comme le sang, noire comme l'ébène! Cette fois, les nains ne peuvent plus te ramener à la vie.” " (Da betrachtete es die Königin mit grausigen Blicken und lachte überlaut, und sprach „weiß wie Schnee, roth wie Blut, schwarz wie Ebenholz! Diesmal können dich die Zwerge nicht wieder erwecken.") Ibid., p. 302.

59. Ibid., p. 303. 
prier m'émut, / et votre visage aussi doux / que neige au baiser du soleil» (Mich rührte Eure holde Bitt') ${ }^{60}$. La ténuité de la neige la rend sensible aux caresses de l'amour, mais cette fragilité menace la jeune fille de disparition. Blanche-Neige reprend elle-même l'image un peu plus loin dans une scène qui l'oppose au Prince, attiré par les charmes de la Reine :

$O$, ich verlange ja nichts mehr,

als daß ich lächelnd tot bin, tot.

Das bin ich auch und war es stets. -

Nie fühltich heißen Lebenssturm.

Ich bin so still wie weicher Schnee, der für den Strahl der Sonne liegt, daßsie ihn nimmt. Schnee bin ich so und fließe bei dem warmen Hauch, der mir nicht, der dem Frühling gilt. Süß ist dies Sickern. Liebe Erd', nimm mich in deine Wohnung auf? So weh ist an der Sonne mir.

$\mathrm{Oh}$, je ne demande plus rien qu'être morte en souriant, morte. Je le suis, l'ai toujours été. Insensible aux vents chauds de vie, je me tais comme tendre neige s'offrant aux rayons du soleil pour qu'il la prenne. Oui, je suis neige et l'haleine chaude où je fonds n'est pas mienne, elle est du printemps. Doux, le suintement. Terre aimée, accueille-moi en ta demeure.

Il me fait si mal, le soleil ${ }^{6 r}$.

Marie-Louise Audiberti, qui voit dans l'emploi de ce motif la marque d'une «distinction particulière [...] réservée aux êtres d'élite» dans l'œuvre de Walser, rapproche "ce raffinement intérieur» d' "une chasteté mortelle». Blanche-Neige, parce qu'associée à la neige, serait "déjà morte au désir ${ }^{62}$ ». La comparaison avec la Reine est éloquente à cet égard : «La luxuriance / du printemps n'est pas si exquise», constate la jeune fille : «Est-ce elle qui devrait m'envier / quand, pareille à l'hiver, je suis / à ses côtés, gelée et froide? 》 (Die üppige Pracht I des Frühlings ist so köstlich nicht; Und sie, sie sollte neidisch

6o. R. Walser, ouvr. cité, p. I2-13.

6I. Ibid., p. 35-37.

62. M.-L. Audiberti, "La neige - Fantasmes de l'effacement chez Robert Walser", dans V. A. Deshoulières (dir.), Effets de neige. L'épopée à l'épreuve du froid, 1998, p. 83-84. 
sein / auf mich, die wie der Winter ihr / frostg und kalt zur Seite steht?') ${ }^{63}$. Stérile est la neige qui touche Blanche-Neige et les métaphores érotiques qui constituent l'armature de cette tirade désignent en creux la sensualité absente de la princesse. Pour Gilbert Durand, la «rêverie neigeuse» serait «rêverie négatrice ${ }^{64}$ »: «Dans l'univers de l'hiver la pensée se recueille et devient angélique, loin des exubérances charnelles de l'étét ${ }^{65}$. "La rêverie matricielle qui donne naissance au personnage éponyme et donc au conte se diffuse dans le drame sous forme de notations récurrentes ${ }^{66}$. L'organisation réticulaire du motif marque le passage du récit au poème en privilégiant l'axe du paradigme et sous-tend la dimension symbolique du texte. Dans la récriture de Robert Walser, Blanche-Neige est bien vivante, nul n'attente à sa vie sinon sous forme de simulacre, mais un secret tropisme l'attire vers la mort. Le dialogue avec le Prince est révélateur de ce fonctionnement :

\author{
Prinz: \\ [...] \\ O, ließ ich dich doch in dem Sarg. \\ Wie schön du darin lagst. So liegt \\ Schnee auf der stillen Winterwelt. \\ Schneewittchen: \\ Schnee, immer Schnee? \\ Prinz: \\ Verzeih, du liebes Winterbild, \\ du Abbild frommer, weißer Ruh'. \\ Le Prince : \\ [...] \\ Que ne te laissai-je au cercueil! \\ Tu y gisais si belle. Ainsi \\ gît la neige en l'hiver muet. \\ Blanche-Neige : \\ Neige, toujours neige?
}

\footnotetext{
63. R. Walser, ouvr. cité, p. 90-9I.

64. G. Durand, «Psychanalyse de la neige», dans D. Chauvin (éd.), Champs de l'imaginaire, 1996, p. 15 .

65. Ibid., p. 20.

66. Le motif de la neige semble primordial pour définir le personnage éponyme dans son essence dans les différentes récritures du conte. Dans le présent volume, Nathalie Prince en montre l'importance chez J. Lorrain dans «La Princesse Neigefleur", qui précède de peu, en France, le texte de Walser. (J. Lorrain, La Princesse Neigefleur [publié isolément dans L'Écho de Paris le 9 janvier I894 sous le titre Conte pour la Nuit des Rois; recueilli dans Sensations et souvenirs, Paris, G. Charpentier et E. Fasquelle, I895, sous le titre "Conte pour la Nuit des Rois»; rassemblé avec les autres contes de J. Lorrain dans la section Contes de givre et de sommeil, dans Princesses d'ivoire et d'ivresse, Paris, Ollendorff, 1902].)
} 


\section{Le Prince :}

Pardon, chère image d'hiver,

pieuse figure, calme et blanche ${ }^{67}$.

La séquence qui montre le «fils de roi» (der Königssohn) séduit par «la belle Blanche-Neige qui était allongée à l'intérieur» du cercueil de verre (das schöne Schneewittchen darin) ${ }^{68}$ s'épure dans ce fragment mémoriel en se focalisant sur un seul des éléments du conte : «elle était toujours aussi blanche que la neige" (es war noch so weiß als Schnee). Le rouge et le noir s'effacent et le portrait se recompose autour d'une symphonie en blanc qui célèbre le «caractère céleste fondamental» de la neige ${ }^{69}$. Or cette idéalisation va de pair avec la représentation très particulière du conte, qui pose un autre temps impliquant "une autre logique du monde ${ }^{70}$ ", la mise en place d'un «monde inactuel, alternatif, concurrent du monde actuel ${ }^{71}$ ». Lorsque Blanche-Neige se remémore son séjour chez les nains, la neige est de nouveau convoquée pour définir un univers "calme et beau": "D'un calme de neige, c'était / la paix" (Still wie der Schnee lag Ruhe dort) ${ }^{72}$. Les objets - table ou drap - sont marqués du sceau de la pureté dans cet espace de la mémoire qui est celui du conte, un «là-bas» (dort) qui s'oppose à un "ici", "chez vous, dans ce monde» (zu Euch, zur Welt zurück). "Là, nul mot ne m'atteignait» (Nie spürtïch dort ein rahes Wort) ${ }^{73}$, confie encore Blanche-Neige, confirmant par là que le conte est régi par ses lois propres. Le dramolet, qui repose précisément sur un passage du conte au drame, met en échec les valeurs prônées par le conte, valeurs dont certains motifs privilégiés se redistribuent dans le texte selon une configuration nouvelle.

\section{La greffe}

L'une des distorsions les plus considérables que Robert Walser fait subir au conte des frères Grimm est, on l'a dit, le rôle attribué au Chasseur, promu

67. R. Walser, ouvr. cité, p. 36-37.

68. Contes pour les enfants et la maison, 2009, t. I, p. 303.

69. G. Durand, art. cité, p. 23.

70. J.-M. Adam, ouv. cité, 20io, p. 242.

7I. Christine Noille-Clauzade, «Le pouvoir de la voix : rhétorique de l'énonciation et statut de la fiction dans l'écriture des contes de fée à la fin du XviI e siècle», dans A. Defrance et J.-F. Perrin (éds), Le Conte en ses paroles. La figuration de l'oralité dans le conte merveilleux du Classicisme aux Lumières, Paris, Desjonquères, p. Io. Cité par J.-M. Adam, art. cité, p. 242.

72. R. Walser, ouvr. cité, p. 74-75.

73. Ibid., p. 76-77. 
au rang de protagoniste, aux côtés de la Reine, de Blanche-Neige et du Prince. Dans le conte, si le Chasseur joue un rôle d'adjuvant en permettant à Blanche-Neige d'avoir la vie sauve, il n'apparaît que dans une seule séquence. Il n'a pas l'étoffe d'un authentique personnage et pas d'identité : «Un jour, elle [la Reine] appela un chasseur près d'elle» (Da rief sie einen Jäger) ${ }^{74}$. Dans le drame, le Chasseur n'est pas seulement présent dans les séances rétrospectives concernant la scène de la forêt; il a son rôle à jouer auprès de la Reine dont il est devenu l'amant tandis que le Roi, toujours vivant, ne se manifeste qu'à la toute fin de la pièce. Or cette audacieuse subversion de l'hypotexte a pour corollaire l'inclusion d'un motif exogène, qui prend l'ampleur d'un leitmotiv structurant, le baiser.

Dès l'ouverture, Blanche-Neige refuse à la Reine un baiser conciliateur à cause de la double perversion que ce signe représente, en l'occurrence prendre un amant et le pousser au crime :

Wie kann ich küssen diesen Mund,

der küssend diesen Jäger trieb

zur rohen Tat. Nie küssich dich.

Moi, baiser cette bouche qui,

baisant le Chasseur, le poussa

à l'acte brutal? Je ne puis ${ }^{75}$.

Tout au long du drame, ce baiser est au cœur des tentatives de restitution du passé, censé correspondre au temps du conte. Accusation ${ }^{76}$, dénégation ${ }^{77}$, revendication ${ }^{78}$ se succèdent en un tourbillon vertigineux jusqu'à pervertir le texte de référence qui perd son autonomie au gré de ces reconstitutions finalement fallacieuses. Il n'est pas jusqu'à la scène où le conte se rejoue à l'intérieur du drame, à la façon d'Hamlet ${ }^{79}$, qui n'altère la séquence originelle par la greffe de ce motif:

Jäger:

Die Kön'gin haßst dich; sie befahl,

74. Contes pour les enfants et la maison, 2009, t. I, p. 297.

75. R. Walser, ouvr. cité, p. I4-I5.

76. R. Walser, ouvr. cité : Blanche-Neige : «Par tes baisers tu enflammas / Le chasseur que voici à tuer" (Mit Küssen feuertest du ja / den Jäger hier zum Töten an), p. I4-I5.

77. Le Chasseur : "Que le feu de ces baisers m'ait / poussé à tuer, ce n'est pas vrai.» (Daßsie mit feurigen Küssen mich/zur Untat antrieb, ist nicht wahr.) Ibid., p. 88-89.

78. La Reine : «Baisers, c'est de baisers que j’ai / enflammé le Chasseur, le tueur. / Ah, pluie de baisers, ah, rosée / sur ce visage» (Mit Küssen, Küssen feuerte / den Jäger ich, den Mordkerl, an. / Ach, wie die Küsse regneten, I wie Tau auf das Gesicht herab), ibid., p. 56-57.

79. On sait que Walser a rêvé d'être acteur de théâtre. «Il dévore alors les grands classiques de Shakespeare à Goethe» (C. Sauvat, Robert Walser, 1989, p. 69). 
hier dich zu töten, heftig trieb sie mich mit süßen Küssen an.

Königin:

$H a, h a$, mit Küssen, ha, ha, ha.

Le Chasseur:

La Reine te hait. Il fallait

qu'ici je te tue; pressante, elle

m'y poussait par de doux baisers.

La Reine :

Ha, ha, des baisers, ha, ha, ha ${ }^{80}$ !

Le baiser est ce sur quoi repose la reconversion du conte en drame poétique. Histoire d'adultère et de désir d'infanticide, le Blanche-Neige de Walser place le sujet face à son insertion obligée dans le monde et pose le problème du mûrissement. "On voit justement grâce à Hamlet, que le mûrissement est une contrainte, donc quelque chose de tout à fait indésirable», écrit Walser dans son Essai sur Hamlet ${ }^{81}$. Le baiser devient le support de cette réflexion, suggérée par la relecture du conte. Blanche-Neige est en effet le témoin indirect de la trahison de la Reine par l'intermédiaire du Prince, lui-même victime du spectacle qu'il contemple : "La Reine aux lèvres du chasseur / donne des baisers qu'il lui rend" (Die Königin küßt des Jägers Mund, / un der gibt Kuß um Kußzurück) ${ }^{82}$. La Princesse se refuse à voir : "Plus que voir, j'aimerais entendre» (Gern, statt zu schauen, hörtich es) ${ }^{83}$, ce qui revient à dire qu'un nouveau conte se substitue à l'ancien, un conte qui donne «la nausée» ([...] es würd'üble mir dabei) ${ }^{84}$ mais avec lequel il faudra bien composer. La Reine explique : "On te loue, / innocente, on me dit mauvaise, / car d'amour j'ai nourri le meurtre, / l'enflammant de baisers fielleux" ([Jedermann] preist dich Unschuld'ge, nennt mich schlecht, / da ich den Mord mit Lieb' gespeist, / ihn feuerte mit gift'gem Kuß ${ }^{85}$. La reconfiguration du conte que permet la mise en abyme repose sur une distorsion du récit merveilleux. Le recours à la méta-énonciation ("On te loue», "on me dit») est le vecteur de ce déplacement : l'antithèse "innocente» (Unschuld'ge) / " mauvaise» (schlecht) désigne le soubassement axiologique sensible dans le conte des frères Grimm, mais la cause alléguée en modifie profondément le sens. Or l'emblème en est le baiser, hyperbolique et véné-

8o. R. Walser, ouvr. cité, p. 64-65.

8I. R. Walser, "Essai sur Hamlet", dans Sur quelques-uns et sur lui-même, I993, p. 74.

82. R. Walser, Blanche-Neige, p. 30-31.

83. Ibid., p. 30-3I.

84. Ibid., p. 32-33.

85. Ibid., p. 8o-8I. 
neux. C'est pourquoi le motif circule dans le drame, suivant des fluctuations qui en exploitent la polysémie.

Le baiser refusé à la Reine à l'ouverture de la pièce scelle l'évolution du personnage en voie de maturation.

Jäger:

[...]

Schneewittchen, küsssie, wenn ich dich

um etwas Liebes bitten darf.

Schneewittchen (küsst sie):

Erlaubt das süße Zeichen mir.

Wie seid Ihr blaß! Verzeiht, wenn ich

mit Küssen dieser Blässe will

das Leben nehmen. Tränken sie

doch alle traurige Farbe auf,

die Eure Wonne so enstellt.

Le Chasseur :

Puis-je t'en prier, Blanche-Neige,

fais-moi plaisir, embrasse-la.

Blanche-Neige (l'embrassant) :

Permettez ce doux témoignage.

Que vous êtes pâle! Pardon,

je veux, par mes baisers, priver

de vie cette pâleur. Qu'ils boivent

toute la funèbre couleur

qui défigure vos attraits ${ }^{86}$.

Cette "thérapeutique du baiser ${ }^{87}$ " procède d'une double inversion : là où le baiser purifie ou guérit par transmission d'un souffle ou d'un fluide curatifs $^{88}$, Blanche-Neige se propose d'absorber le mal dont souffre la Reine. Ce vampirisme à rebours - puisqu'il s'agit de redonner vie à la Reine en lui ôtant tout remords - témoigne de la métamorphose de BlancheNeige qui, absorbant un surcroît de "pâleur», celle que cause la culpabilité, surdétermine sa blancheur native par une forme de connaissance de la Faute et du Pardon, du Bien et du Mal. Le drame s'achève du reste sur un enchaînement de baisers de paix : de Blanche-Neige au Roi, son père, figure de réconciliation, de Blanche-Neige à la Reine et du Roi à Blanche-Neige.

86. Ibid., p. 94-97.

87. Voir sur ce point A. Montandon, Le Baiser. Le corps au bord des lèvres, 2005, p. 35.

88. A. Montandon évoque à ce propos le "caractère sacré» du baiser et «la puissance dont il est investi pour transmettre le souffle vital» (ibid.). 
On note toutefois une absence dans ce ballet final : le baiser du Prince à la Princesse, pourtant évoqué par deux fois comme un possible du conte. La Reine en propose une version légèrement biaisée :

Königin:

Den gift'gen Apfel schickt' ich dir;

du aßest ja davon und starbst.

Dich trugen Zwerge in dem Sarg,

dem gläsernen, bis auf den Kuß

des Prinzen du lebendig wardst.

Es ist doch alles so, nicht war?

Schneewittchen:

Bis auf den Kuß ist alles wahr.

Hier diese Lippen Küßte nie

noch ein entweihnder Männermund.

[...]

Von einem Prinzenkusse sagt

nichts mehr, Mama. Der Kuß ist tot,

als hätt' er nie das Naß gespürt

beidseitigen feuchten Lippenpaars.

La Reine :

Pomme et poison venaient de moi :

tu y goûtas, tu en mourus.

Tu fus mise au cercueil de verre

par les nains, jusqu'à ce baiser

du Prince qui te fis revivre.

C'est bien cela, n'est-il pas vrai?

Blanche-Neige :

À part le baiser, tout est vrai.

Ces lèvres, jamais bouche d'homme

ne les profana d'un baiser.

[...]

D'un baiser du Prince, ne dites

plus mot, Maman. Mort, le baiser :

n'aurait-il donc jamais senti

couple de lèvres deux fois moites ${ }^{89}$ ?

Le correctif apporté par Blanche-Neige a double effet. Il remet le récit en conformité avec le conte des Grimm, où il n'est en effet pas question de baiser. Blanche-Neige s'éveille dans des conditions sensiblement différentes selon que l'on considère la version de I8I2 ou les versions suivantes, mais à chaque fois c'est un choc qui la libère du «morceau de pomme empoi-

89. R. Walser, ouvr. cité, p. 50-53. 
sonnée» (der giftige Apfelgrütz) ${ }^{90}$ coincée dans sa gorge. Mais ici la Princesse interprète cette absence comme le signe d'une défaillance imputable à la fois à sa propre chasteté et à l'inexpérience du Prince.

La fin du drame toutefois est plus ambiguë si l'on se place dans la perspective de la récriture. Blanche-Neige ayant finalement accepté les contingences de l'existence et les faiblesses de l'époux, la Reine avance une conclusion : "je dirai - que dirai-je? Ah oui, je dirai, à la manière du hasard : "Par tes baisers, tu l'enflammas..."” ([...] sag' — Was sage ich? Ach ja, sag'dann, I so wie der Zufall etwa sagt: I "Du feuertest mit Küssen ihn / zu dem... " 9 9. Le réemploi de l'expression utilisée pour désigner sa propre passion adultérine ${ }^{92}$ peut être compris comme l'affirmation d'une filiation enfin acceptée, chacun prenant sa place dans la ronde des passions. Cependant, l'antéposition emphatique marque la pseudo-citation au coin du cliché. C'est pourquoi Blanche-Neige, enfin sortie de l'univers merveilleux des contes, refuse la formule en ces termes : «Oh, taisez-vous. Seul le conte a / dit ces mots — vous, non, moi, jamais" (Schweigt doch, o schweigt. Das Märchen nur / sagt so, nicht Ihr und niemals ich) ${ }^{93}$. Or le syntagme "das Märchen» désigne tout au long du drame le conte des frères Grimm, Blanche-Neige ${ }^{94}$. Mais, greffant un motif exogène dans sa réécriture, Robert Walser combine entre eux et reconfigure deux contes distincts : c'est à "Rose d'épine» (Dornröschen) qu'il emprunte la scène du baiser, également absente de «La Belle au Bois Dormant» des Histoires ou contes du temps passé de Perrault. Ce conte, qui reprend la première partie du récit de Perrault, propose en effet une variation sur l'éveil de la Princesse. Là où, chez Perrault, le prince s'agenouille et assiste à son éveil programmé9s, chez Grimm, «il se pencha et lui donna un baiser» : "Aussitôt que ses lèvres l'eurent effleurée, Rose d'épine ouvrit les yeux, se réveilla et le regarda très gentiment» ([...] und er bückte sich und gab ihm einen Kuß. Wie er es mit

90. Contes pour les enfants et la maison, 2009, t. I, p. 304.

91. R. Walser, ouvr. cité, p. Io9.

92. "Je l'ai enflammé de baisers" (Ich feuerte mit Küssen ihn), ibid., p. 80-8r.

93. Ibid., p. 108-109.

94. Par exemple : «Ne crois pas le conte aberrant / qui verse à l'ouïe du monde avide / la nouvelle que je suis folle / d'envie» (Ach, glaub' doch / solch aberwitzigem Märchen nicht, I das in der Welt begierig Ohr / die Nachricht schüttet, ich sei toll / aus Eifersucht), p. I8-19, ou encore : La Reine : "Mais le conte / le dit : moi, la Reine mauvaise, / je t’ai envoyé le Chasseur» (Das Märchen ja I sagt, daß ich schlimme Kön'gin sei, / daß ich den Jäger dir gesandt), p. 52-53; Le chasseur : "Beauté n'a pas, pour la beauté, / La haine que prétend le conte" (Schönheit haßt Schönheit nicht so sehr, I wie Märchen es hier ausgesprengt), p. 90-9I.

95. «Alors, comme la fin de l'enchantement était venue, la Princesse s'éveilla [...]» (Charles Perrault, Contes, p. 252). 
dem Kuß berührt hatte, schlug Dornröschen die Augen auf, erwachte, und blickte ihn ganz freundlich an ${ }^{96}$. Cette greffe permet à Walser de fondre deux contes de prédilection, mais elle se redouble d'une inversion, puisque dans cette variante inédite c'est Blanche-Neige qui «enflamme» (feuert) le Prince de ses baisers et non l'inverse.

La connotation passionnelle et érotique du verbe suffirait à modifier la perspective, mais l'échange des acteurs contribue in fine à poser comme hypotexte un hybride étonnant, ne renvoyant ni à Blanche-Neige, ni à Rose d'épine, mais plus probablement à un épisode des aventures de Psyché telles que les relate Apulée dans L’Âne d'or. Dans le livre VI, Psyché se pique le doigt avec l'une des flèches du carquois de son mari : "C'est ainsi que l'innocente Psyché, d'elle-même, est devenue amoureuse de l'Amour ${ }^{97}$.» Le narrateur poursuit : "Alors, enflammée, à chaque instant davantage, de désir pour l'Amour, elle se penche sur lui, pleine de passion, lui donne, aussi vite qu'elle peut, des baisers ardents $[\ldots]^{98}$.» On reconnaît là le schéma comme le lexique que réemploie Walser. Ute Heidmann a mis en lumière de façon très convaincante l'écriture "palimpseste» des contes (de Perrault et des Grimm, notamment) et souligné le rôle majeur que joue le conte (fabella) de Psyché99. Cette histoire - dont on peut par ailleurs penser qu'elle sous-tend en grande partie le conte Blanche-Neige — pourrait également avoir fourni à Walser une source intéressante. Cet épisode fort connu en effet précède immédiatement la séquence où Cupidon, brûlé par la goutte d'huile, s'enfuit loin de son épouse. L'ardeur amoureuse, loin de sceller la passion, en provoquerait la disparition, inversant la leçon du conte.

La fabrique à l'œuvre dans le dramolet en vient donc, à force de distorsions, à substituer à un référent déjà flottant (de quelle version du conte des frères Grimm s'agit-il ?) un référent multiforme. Blanche-Neige toutefois le rejette, libérant ainsi son personnage de toute attache intertextuelle. Le motif du baiser permet alors d'articuler l'hypotexte et l'ensemble de variations auxquelles il est soumis avec le dramolet qui évacue peu à peu l'histoire dont il se nourrit et en poétise les composantes majeures.

C'est dans cette optique que Blanche-Neige oppose le "penser» au «sentir ${ }^{100} »$. Pour elle, la rectitude stérile $d u$ "penser» repose sur la prévalence

96. Contes pour les enfants et la maison, 2009, t. I, p. 284.

97. Apulée, L'Âne d'or ou les métamorphoses, 1985, p. 130.

98. Ibid., p. I30. Je souligne.

99. Voir les chapitres "Le Petit Chaperon rouge palimpseste» et "La Barbe bleue palimpseste», dans U. Heidmann et J.-M. Adam, Textualité et intertextualité des contes, 20IO, p. 8I-III et II3-I52, ainsi que, pour La Belle au bois dormant, "Expérimentation générique et dialogisme intertextuel», $20 I I$. IOO. "Sentir pense vif» (Gefühl denkt scharf), p. 48-49; R. Walser, ouvr. cité. 
des «faits» et des "opinions", système à l'œuvre dans toute une partie du dramolet, quand les personnages s'avisent de juger ce qu'ils croient avoir été les actes exposés impitoyablement dans le conte. En revanche, le «sentir» autorise une complète reconfiguration, qui est libération. Écoutons BlancheNeige parlant du «sentiment» (Gefühl) :
hört, was es sagt. $O$, es sagt nichts.
Es lächelt, küßt die Sünde tot,
liebkost als seine Schwester sie, erwügt sie küssend.
Écoutez : que dit-il? Oh, rien, il sourit, il donne au péché un baiser mortel, le caresse en frère et, l'embrassant, l'étrangle ${ }^{\text {Ior }}$.

Le sentiment libère des conventions, et c'est encore le motif du baiser, le plus étranger au texte de référence mais le plus communément associé au conte dans l'esprit des lecteurs qui, allégorisé, porte poétiquement ce principe. Baiser curatif ou de conciliation, baiser érotique et d'éveil aux sens, convergent vers ce «baiser mortel» qui inverse radicalement le topos pour conjurer l'emprise des conventions et de la parole immuable, trop longtemps rapportée. Si l'on suit la leçon du chasseur, il ne reste plus qu'à se taire : «La fin s'embrasse dans la fin, / sans que le début soit fini» (End küßt sich in dem End, wenn auch / Anfang noch nicht zu Ende ist) (p. 97). À la clôture du conte s'oppose l'ouverture féconde du drame poétique qui le défait pour le refaire autrement, sans penser à poser jamais le point final. C'est bien plutôt un suspens dont le dramaturge préconise ici l'usage, ce qui l'éloigne de la clôture trop radicale du conte.

Dans le Schneewittchen de Walser, le conte n'est pas un personnage comme c'est le cas dans son Aschenputtel (Cendrillon). Il n'en est pas moins présent dans ce dramolet qui en rappelle sans cesse le souvenir imposant : «le conte / l'a, haut et vrai, bien fait savoir" (wie laut und wahr / das Märchen ja verkündet hat), affirme le Chasseur dès l'ouverture de la pièce ${ }^{102}$. Que le drame soit enfanté par le conte n'est pas contestable, qu'il donne lieu à réflexion ne l'est pas moins, que la reconfiguration que propose Robert Walser repose sur la contestation est tout aussi flagrant. Les commentaires en effet se multiplient, dans ce «métathéâtre». Certes, le conte «impose»

IOI. R. Walser, ouvr. cité, p. 50-5I.

IO2. R. Walser, ibid., p. I2-I3. 
sa leçon ${ }^{103}$, mais il faiblit tout au long des échanges et des tentatives de reconstitution qui reposent sur un jeu théâtral qui est aussi jeu avec le sens, comme l'attestent ces paroles de la Reine :

[...] Ach, glaub' doch

solch aberwitzigem Märchen nicht,

das in der Welt begierig $\mathrm{Ohr}$

die Nachricht schüttet, ich sei toll

aus Eifersucht, bös von Natur,

was alles ein Geschwärtz nur ist.

$\mathrm{Ne}$ crois pas le conte aberrant

qui verse à l'ouïe du monde avide

la nouvelle que je suis folle

d'envie, et naturellement

mauvaise : rien que bavardages ${ }^{104}$.

$\mathrm{Au}$ conte ancien doit se substituer un conte nouveau; le dramaturge incite à ne plus se tourner vers «la vieille porte» (die alte Tür) ${ }^{105}$, mais à aller de l'avant. Le chemin, frayé pour dégager ce sens nouveau, qui reste instable, n'est pas simple. Il est fait de méfiance, de regrets, de revirements. Blanche-Neige la première demande une autre histoire ${ }^{\mathrm{Io} 6}$ : "Tenez-vous tant à ce tracé / que vous le dessiniez toujours?" (Hangt Ihr an diesen Zügen so, I daß Ihr sie immer zeichnen mü $\beta$ t? ${ }^{107}$. Elle en propose des versions successives, qui font tourner les personnages dans une danse folle : le Prince est «versatile / comme un roseau qui çà et là / s'agite» (Woher die schwache Sinnesart, / die wie ein Schilfrohr hin und her, [...] sich bewegt?) ${ }^{\text {108 }}$, le canevas de la pièce est fait de "voltes ${ }^{109}$ ", le sens s'infléchit et se distord : "que de non-sens en ces sens!» (Ei, welcher Unsinn ist im Sinn $)^{\text {Iо }}$, l'inadéquation du signifiant et du signifié triomphe : «L'amour est muet soupçon, haine. /

I03. La Reine évoque le geste horrible "que le conte impose pour fond / à l'action que nous jouons ici» (wie Märchen sie zugrunde legt / hier dieser Handlung, diesem Spiel), ibid., p. I8-I9.

I04. Ibid., p. I8-19.

I05. Blanche-Neige s'adresse au Prince qui se détourne d'elle en ces termes : «Je crie : Blanche-Neige t'attend, / viens frapper à la vieille porte" ([Ich rufe] Schneewittchen wartet dir, / komm, klopfe an die alte Tür), ibid., p. 40-4I.

I06. "Ah, je la connais, cette histoire, / [...] par bonté, contez-m'en une autre» (Ah, die Geschichte kenn' ich ja, / [...] Erzählt was anders, seid so gut), ibid., p. 54-55.

I07. Ibid., p. 56-57.

I08. Ibid., p. 6o-6I.

I09. "Voici que, par une autre volte, / le Prince te rend son amour" (Der Prinz ja wieder wandte sich / von neuem dir in Liebe zu), ibid., p. 72-73.

IIo. Ces propos de Blanche-Neige sont à double entente. Il s'agit des sens du Prince attiré par la Reine, mais le contexte autorise une double lecture (ibid., p. 40-4I). 
Le Prince? Un chasseur. La vie : mort» (Lieb'ist argwöhn'scher, stummer Haß. / Prinz ist ein Jäger, Leben Tod) "'. Le jeu mené par les protagonistes est "jeu branlant» (dieses lockre Spiel) ${ }^{\text {I2 }}$, le " oui » et le "non" s'affrontent et s'épousent ${ }^{\mathrm{Ir} 3}$. Le métathéâtre de Walser conclut à la fausseté du conte en sa certitude : "C'est un mensonge noir et fou, / Dur à entendre, bon à faire / Peur aux enfants" ('s ist eine Lüge schwarz und toll, / widrig zum Anhörn. Kindern macht / man bang damit) ${ }^{\mathrm{II}}$. C'est l'essence même du genre qu'il s'agit de revisiter.

Mais les jeux de la récriture, qui deviennent eux-mêmes objets de l'écriture, ont une seconde finalité : transformer le dit du conte en matière poétique. Le canevas narratif oscille et se défait, le statut des personnages vacille, le texte de référence lui-même s'estompe, sujet au brouillage et support de greffes multiples, mais corrélativement un processus de poétisation est à l'œuvre. C'est lui qui procède, dans la complexe alchimie des transmutations intertextuelles, à une structuration paradigmatique du texte, dont les fluctuations se trouvent transcendées par cette mutation du conte en Poème ${ }^{\mathrm{II}}$.

\section{Bibliographie}

Apulée, L'Âne d'or ou les métamorphoses, trad. Pierre Grimal, Paris, Gallimard, coll. «Folio», I985.

Audiberti Marie-Louise, "La neige - Fantasmes de l'effacement chez Robert Walser", dans V. A. Deshoulières (dir.), Effets de neige. L'épopée à l'épreuve du froid, Clermont-Ferrand, Cahiers du CRLMC, I998, p. 83-84. Contes pour les enfants et la maison, collectés par les frères Grimm, édités et traduits par Natacha Rimasson-Fertin, Paris, José Corti, 2009, t. I.

Durand Gilbert, "Psychanalyse de la neige», dans D. Chauvin (éd.), Champs de l'imaginaire, Grenoble, Ellug, 1996.

Harrer Konrad, Souveraineté et impuissance dans l'ouvre de Robert Walser, Berne, Peter Lang, coll. «Contacts», 2008.

\footnotetext{
III. Ibid., p. 78-79.

II2. Ibid., p. 82-83.

II3. Voir en particulier la réponse de Blanche-Neige au Chasseur : «Oui, et pourtant non. Si j'étouffe / le oui, prompt le non redit oui" (Ja und doch nein. Erwürg' ich ja, / sagt nein mir wieder hurtig ja), ibid., p. 84-85.

II4. Ibid., p. 94-95.

II5. Tel était l'avis de R. Walser lui-même, écrivant à Ernst Rowoht : "Cendrillon et Blanche-Neige sont entièrement Poésie [...]. Elles visent le style et la beauté; l'essentiel dans ce cas est le plaisir qu'on prend au livre [...]. Elles sont accordées pour la parole et la langue, pour la mesure et le plaisir du rythme.» (Cité dans R. Walser, Blanche-Neige, 4 e de couverture.)
} 
Heidmann Ute, «Expérimentation générique et dialogisme intertextuel : Perrault, La Fontaine, Apulée, Straparola, Basile», Féeries, nº 8, 20II, p. 45-69.

Heidmann Ute et Adam Jean-Michel, Textualité et intertextualité des contes, Paris, Classiques Garnier, 2010.

Montandon Alain, Le Baiser. Le corps au bord des lèvres, Paris, Éd. Autrement, 2005.

Palacio Jean de, Les Perversions du merveilleux, Paris, Séguier, I993.

Perrault Charles, Contes, Paris, GF-Flammarion.

SAuvat Catherine, Robert Walser, Paris, Plon, I989.

UTz Peter, Robert Walser : danser dans les marges, Carouge-Genève, Éd. Zoé, [1998] 200I (pour la version française).

Walser Robert, "Essai sur Hamlet», dans Sur quelques-uns et sur lui-même, Gallimard, 1993.

—, Blanche-Neige, traduit par Hans Hartje et Claude Mouchard, bilingue, Paris, José Corti, coll. «Merveilleux, nº I8», 2006. 